\title{
Pulsatile ocular blood flow: the effect of the Valsalva manoeuvre in open angle and normal tension glaucoma: a case report and prospective study
}

\author{
J C Khan, E H Hughes, B D Tom, J P Diamond
}

Br J Ophthalmol 2002;86:1089-1092

Aim: A case of severe normal tension glaucoma is reported in a trumpet player, along with a study investigating the association between glaucoma and raised intrathoracic pressure, using measurements of pulsatile ocular blood flow (POBF).

Methods: Three patient groups were studied; normals ( $\mathrm{n}=$ 34), untreated primary open angle glaucoma (POAG) ( $n=$ 20), and untreated normal tension glaucoma (NTG) ( $n=$ 22), with a total of 76 patients who underwent measurements of $\mathrm{POBF}$ using the $\mathrm{OBF}$ pneumotonometer at rest and while forcibly exhaling through a mouthpiece connected to a mercury manometer $(30 \mathrm{~mm} \mathrm{Hg})$ (Valsalva manoeuvre).

Results: POBF fell during Valsalva in all groups with the greatest predictor being the resting value of POBF. There was no evidence of significant differences in the mean change in $\mathrm{POBF}$ occurring during the Valsalva manoeuvre for the three groups studied before or after adjusting for the sex, the resting POBF, and the resting IOP of the patients $(\mathrm{p}$ $=0.294$ and $p=0.542$, respectively). However, statistically significant associations were found between the change in POBF and sex $(p=0.049)$, resting POBF $(p<0.0001)$ and resting IOP $(p=0.032)$. Males had a greater drop, on average, in POBF during Valsalva manoeuvre than females after adjusting for the other factors. Additionally, there was a significant difference in the mean change in IOP during Valsalva for the three groups $(p=0.002)$, with the difference occurring between the normal and POAG groups $(p<0.005)$. The POAG group had, on average, a drop in IOP during Valsalva, while the other two groups had an increase in IOP. Also noted was a significant difference in the distributions of the risk factors among the three groups $(p=0.002)$.

Conclusions: This study demonstrates no difference between groups with respect to resting or Valsalva POBF, but does demonstrate a possible trend with respect to IOP, with a drop in IOP occurring during Valsalva in the POAG group. There is, however, much variability in the data left unexplained by our models. Thus, unfortunately, we cannot advise our trumpet player whether his NTG is directly related to his trumpet playing.

A 60 year old man presented with optic disc cupping, and visual field defects (right eye; vertical cup:disc ratio 0.4, mean defect $-3.55 \mathrm{~dB}$, left eye; vertical cup:disc ratio 0.7 with an inferior notch, mean defect $-16.26 \mathrm{~dB}$ ). Intraocular pressures were $17 \mathrm{~mm} \mathrm{Hg}$ in each eye and mean resting pulsatile ocular blood flow (POBF) at rest was right eye $947 \mu \mathrm{l} / \mathrm{min}$; left eye $812 \mu \mathrm{l} / \mathrm{min}$. He reported that he was a professional trumpet player and inquired whether he could continue play- ing for 5 hours each day. While trumpet blowing the following single readings were obtained, right eye $515 \mu \mathrm{l} / \mathrm{min}$; left eye $849 \mu \mathrm{l} / \mathrm{min}$.

The playing of high resistance wind instruments such as the trumpet involves expiration against resistance, mimicking the Valsalva manoeuvre. We designed this study to investigate the effect of the Valsalva manoeuvre upon POBF in normal patients, and untreated patients with primary open angle glaucoma (POAG) or normal tension glaucoma (NTG) to ascertain whether raised intrathoracic pressure could be aetiologically linked with glaucoma and, more specifically, NTG.

\section{PATIENTS AND METHODS}

The study had local ethics committee approval and informed consent was given by study participants. Subjects were recruited sequentially from the new patient glaucoma clinic and were grouped after examination by a single clinician consultant glaucoma specialist (JPD). Glaucoma patients were defined by the presence of typical glaucomatous optic disc cupping plus glaucomatous visual field defects. POAG was confirmed where the IOP was $22 \mathrm{~mm} \mathrm{Hg}$ or more, while NTG diagnosed where the intraocular pressure (IOP) was less than $22 \mathrm{~mm} \mathrm{Hg}$ (Goldmann applanation tonometry). Controls had normal optic discs, full visual fields, and normal $(<22 \mathrm{~mm}$ $\mathrm{Hg}$ ) IOP. Each patient was questioned about specific risk factors: "atherosclerotic" risk factors included ischaemic heart disease (excluding angina as this may be a vasospastic phenomenon in some patients) and hypertension. "Vasospastic" risk factors included Raynaud's phenomenon and migraine. Blood pressure was recorded at rest and heart rate was recorded both at rest and during Valsalva. No patients were on glaucoma treatment. Patients with over 6 dioptres of ammetropia were excluded.

POBF was measured using the POBF tonometer (OBF Labs, UK). The worse affected eye was selected of patients in the glaucoma groups, while controls had an eye selected at random (toss of a coin). Two readings were recorded with the patient seated at rest. The patient was then asked to exhale into a mouthpiece connected to a mercury manometer, maintaining an expiratory pressure of $30 \mathrm{~mm} \mathrm{Hg}$ while two further POBF readings were taken (typically a period of 20 seconds). For data analysis an average of each set of two readings was used.

Univariate comparisons of continuous and categorical variables with group were performed using a one way analysis of variance and Fisher's exact test, respectively. Additional investigations of resting POBF and change in POBF with age and sex was performed using independent sample $t$ test and Pearson's correlation coefficients. Multiple linear regression, using a backward elimination approach, was performed to investigate the effect of group on the change in POBF and the change in IOP after adjusting for variables that were statistically significant in the final model. All analyses were performed using 
Table 1 Patient characteristics and baseline measurements in normal, NTG, and POAG groups

\begin{tabular}{|c|c|c|c|c|}
\hline & Normal & NTG & POAG & p Value \\
\hline Total & 34 & 22 & 20 & \\
\hline \multicolumn{5}{|l|}{ Sex distribution } \\
\hline Male $(n=44)$ & 23 & 12 & 9 & \multirow[t]{2}{*}{ NS } \\
\hline Female $(n=32)$ & 11 & 10 & 11 & \\
\hline \multicolumn{5}{|l|}{ Numbers in each age group } \\
\hline$<60$ years $(n=32)$ & 22 & 5 & 5 & \multirow[t]{2}{*}{0.002} \\
\hline$>60$ years $(n=44)$ & 12 & 17 & 15 & \\
\hline Mean age (SD), years & $54.1(11.8)$ & $65.4(11.2)$ & $66.8(12.5)$ & 0.0002 \\
\hline Mean resting pneumotonometer IOP (SD) $(\mathrm{mm} \mathrm{Hg})(\mathrm{n}=76)$ & $16.4(4.3)$ & $15.6(4.0)$ & $23.7(4.9)$ & $<0.00001$ \\
\hline Mean resting Goldmann IOP (SD) $(\mathrm{mm} \mathrm{Hg})(\mathrm{n}=76)$ & $18.1(2.8)$ & $15.8(2.8)$ & $26.5(3.1)$ & $<0.00001$ \\
\hline Mean resting POBF (SD), $(\mu \mathrm{l} / \mathrm{min})(\mathrm{n}=75)$ & $1086.9(403.4)$ & $1020.0(356.0)$ & $802.1(509.4)$ & NS \\
\hline Mean resting heart rate (SD), $(\mathrm{bpm})(\mathrm{n}=76)$ & $72.1(11.1)$ & $66.0(8.4)$ & $73.4(12.5)$ & NS \\
\hline Mean arterial pressure at rest (SD), $(\mathrm{mm} \mathrm{Hg})(\mathrm{n}=74)$ & $105.8(10.7)$ & $105.0(13.3)$ & $106.7(11.7)$ & NS \\
\hline \multicolumn{5}{|l|}{ Number of patients with specific risk factors $(n=76)$} \\
\hline None & 22 & 7 & 10 & \multirow[t]{4}{*}{0.002} \\
\hline Vasospastic alone & 5 & 9 & 1 & \\
\hline Atherosclerotic alone & 1 & 3 & 8 & \\
\hline Both & 6 & 3 & 1 & \\
\hline \multicolumn{5}{|l|}{ Numbers taking cardiovascular medications } \\
\hline No & 28 & 18 & 13 & \multirow[t]{2}{*}{ NS } \\
\hline Yes & 6 & 4 & 7 & \\
\hline \multicolumn{5}{|l|}{ Refractive error (numbers of subjects) } \\
\hline myopia >-2 D & 4 & 2 & 1 & \multirow[t]{3}{*}{ NS } \\
\hline$-2 D$ to $+2 D$ & 26 & 15 & 16 & \\
\hline hypermetropia $>+2 D$ & 3 & 4 & 1 & \\
\hline
\end{tabular}

NS = non-significant at $5 \%$ level

Note: refer to text for explanation of "adjusted" $p$ values.

the statistical package spss version 9. Statistical significance was assigned at the $5 \%$ level.

\section{RESULTS}

The patients' characteristics for the three groups at baseline are shown in Table 1. No significant difference was found in resting POBF when comparing the three groups. Statistically significant differences between the three groups were, however, found for the mean age $(p<0.001)$, mean resting IOP $(\mathrm{p}<0.001)$, and in the distribution of risk factors $(\mathrm{p}=0.002)$. The difference in age distributions was mainly between the normal and the other two groups, with a tendency towards younger subjects (less than 60 years old) in the normal group. The mean resting intraocular pressure was significantly higher in the POAG group than in the other two groups.

Our risk factor variable was divided into four categories: ( 1 ) no vasospastic or atherosclerotic risk factors; (2) only vasospastic risk factors; (3) only atherosclerotic risk factors; (4) both types of risk factors present. The Fisher's exact test showed that there was an association between type of risk factor and group (Table 1) with neither risk factor being most prevalent in the normal group; vasospastic risk factors being most prevalent in the NTG group and atherosclerotic risk factors most prevalent in the POAG group.

Table 2 shows a subanalysis of POBF in relation to sex, age, and refractive error. The mean resting POBF was inversely correlated with age (Pearson's correlation $-0.324, \mathrm{p}=0.005$ ). If age is categorised into two groups $(<60$ years and $60+$ years) the independent sample $t$ test for comparing the mean resting POBF with age also gave a statistically significant $p$ value of 0.045 . No significant correlation/association was found between age and the change in POBF $(p=0.998)$. Females were found to have a significantly higher resting POBF than males $(p=0.028)$. There was no significant difference among the emmetropes, myopes, and hypermetropes in either resting or change in POBF after adjusting for resting POBF if taken as three refractive error groups; however, there is a trend shown towards a greater change in POBF from myopes through emmetropes to hypermetropes.

Table 3 illustrates the effect of Valsalva on heart rate, IOP, and POBF in our three patient groups. When a multiple linear regression model for the change in POBF (always controlling for group) was fitted with backward elimination employed to remove non-significant variables from the model, the final model that resulted included sex $(p=0.049)$, resting POBF $(p<0.0001)$, and resting IOP $(p=0.032)$, in addition to the group effect. No statistically significant differences were found in the mean change in POBF among the three groups after adjusting for the sex, the resting POBF, and the resting IOP of the patients (Table $3, \mathrm{p}=0.542$ ).

From the above multiple linear regression model it was found that males had a greater drop, on average, in POBF during the Valsalva manoeuvre than females after adjusting for

Table 2 Subanalysis of POBF in relation to sex, age, and refraction

\begin{tabular}{|c|c|c|c|c|}
\hline & $\begin{array}{l}\text { Mean resting POBF (SD) } \\
(\mu \mathrm{l} / \mathrm{min})\end{array}$ & $\mathrm{p}$ Value & $\begin{array}{l}\text { Mean fall in POBF with } \\
\text { Valsalva (SD) }(\mu \mathrm{l} / \mathrm{min})\end{array}$ & $p$ Value \\
\hline Males & $871.2(301.5)$ & 0.028 & $312.0(340.2)$ & 0.038 (adjusted) \\
\hline Females & $1077.4(409.2)$ & & $328.1(291.3)$ & \\
\hline$<60$ & 1107.7 (486.5) & 0.005 & $340.57(284.5)$ & NS (adjusted) \\
\hline$>60$ & $906.2(371.4)$ & & 298.35 (349.2) & \\
\hline Myopes (<-2D) & 770.7 (182.2) & NS & $655.2(346.5)$ & NS (adjusted) \\
\hline Emmetropes $(-2$ to $+2 D)$ & $1047.3(464.8)$ & & $675.0(746.3)$ & \\
\hline Hypermetropes $(.+2 D)$ & $1227.6(452.2)$ & & $1062.4(463.7)$ & \\
\hline
\end{tabular}

NS = non-significant at $5 \%$ level

Note: refer to text for explanation of "adjusted" p values. 
Table 3 Effect of Valsalva on heart rate, IOP, and POBF

\begin{tabular}{lllll}
\hline & Normal & NTG & POAG & $p$ Value \\
\hline Mean Valsalva heart rate (SD), $(\mathrm{bpm})(\mathrm{n}=60)$ & $82.3(15.4)$ & $71.6(10.8)$ & $82.9(14.2)$ & 0.025 \\
Mean change in heart rate (SD), $(\mathrm{bpm})(\mathrm{n}=60)$ & $10.3(11.6)$ & $6.6(7.1)$ & $11.3(9.6)$ & $\mathrm{NS}$ \\
Mean Valsalva IOP (SD), $(\mathrm{mm} \mathrm{Hg})(\mathrm{n}=61)$ & $18.1(4.0)$ & $16.5(5.1)$ & $22.7(5.6)$ & 0.001 \\
Mean change in IOP (SD) $(\mathrm{mm} \mathrm{Hg})(\mathrm{n}=61)$ & $+2.1(2.4)$ & $+0.9(2.5)$ & $-0.6(1.8)$ & 0.002 \\
Mean Valsalva POBF (SD) $(\mu \mathrm{l} / \mathrm{min})(\mathrm{n}=58)$ & $773.4(221.9)$ & $739.7(328.1)$ & $537.8(512.1)$ & $\mathrm{NS}$ \\
Mean fall in POBF (SD), $(\mu \mathrm{l} / \mathrm{min})(\mathrm{n}=58)$ & $375.0(311.0)$ & $221.0(259.5)$ & $334.7(374.4)$ & $\mathrm{NS}$ \\
& & & & $(0.294$ unadjusted \\
& & & & 0.542 adjusted) \\
\hline
\end{tabular}

NS = non-significant at $5 \%$ level.

Note: refer to text for explanation of "adjusted" p values.

the other factors in the model (Table 2, p $=0.038$ ). Also apparent was that the higher the resting POBF the larger the drop in POBF during Valsalva, most likely indicating a regression to the mean effect. Furthermore, on average, increases in resting IOP result in larger drops in the POBF during Valsalva. This regression model only explained about $51 \%$ of the variation in the data.

The final linear regression model for the change in IOP resulted in only the group variable remaining in the modelthat is, the effects of the age, sex, resting IOP, resting POBF, etc, were all eliminated from the final model. It was found that there was a statistical difference in the mean change in IOP among the three groups $(p=0.002)$, with the POAG group having, on average, a drop in intraocular pressure during the Valsalva manoeuvre, while the other two groups had, on average, a rise (see Table 3). Only $19 \%$ of the variability in the change in IOP data was explained by our model using the group effect.

\section{DISCUSSION}

Pneumotonometric methods are used to estimate pulsatile ocular blood flow on the basis of changes in the measurements of intraocular pressure during the cardiac cycle ${ }^{1}$ and provide a non-invasive, continuous measure of the IOP, which, when related to the rate of change of IOP, is used to estimate the pulsatile component of ocular blood flow. ${ }^{2}$

The Valsalva manoeuvre is a standard stimulus for the investigation of cerebral and ocular blood flow in humans. ${ }^{3}$ There are four phases to the Valsalva manoeuvre. ${ }^{4}$ Phase 1 , the initiation of Valsalva, creates a rise in arterial pressure as blood is squeezed from the thoracic aorta. In phase 2, the raised intrathoracic pressure reduces venous return via the vena cava causing a fall in mean arterial pressure (mAP), but still remaining higher than the resting mAP because a reflex tachycardia and peripheral vasoconstriction limits the fall in mAP. On release of the glottis (phase 3) the drop in intrathoracic pressure restores venous return and the mean arterial pressure rebounds rapidly. There is a reflex bradycardia to counter this (phase 4). In normal subjects it has been shown that the pulsatile ocular blood flow is significantly reduced during Valsalva and is proportional to the rise in intraocular pressure. $^{5}$ Since patients with low tension glaucoma develop optic nerve damage at low intraocular pressures we might have expected the drop in POBF during Valsalva to be exaggerated in this group

Previous authors have investigated the association between wind instrument playing, intraocular pressure, and glaucoma. Schuman measured IOP by pneumotonometry while the musicians played wind instruments. Life hours of high resistance wind instrument playing had a significant association with abnormal visual field. ${ }^{6}$

As other workers have found, we noted a marked effect of Valsalva on $\mathrm{IOP}^{7}$ and there was a significant difference between our groups in this effect. In this study, Valsalva phase 2 is artificially prolonged to allow for acquisition of data. We can thus understand the rise in IOP as being mainly a result of raised episcleral pressure reducing aqueous outflow. The engorged choroidal vessels may also produce a small increase in the total ocular volume, which, because of some rigidity of the wall of the eye, results in an increase in IOP. The drop in IOP in the POAG group is less easily explained. It may be a function of the fact that the already high IOP limits the effect of raised episcleral pressure. Also aqueous in-flow by ultrafiltration at the ciliary body may be compromised, causing IOP to fall. Lacey and Rankin, ${ }^{7}$ in their study of normal subjects, noted a difference in the IOP response during Valsalva with respect to age: tending to rise in younger subjects but fall in older subjects. However, by eliminating the effect of age in our groups we still found a difference among our groups.

We demonstrated a trend of a lower resting POBF in our group of POAG subjects, but similar resting POBF in our NTG and normal groups. The normals had a slightly higher but non-statistically different resting POBF than the NTG group. Several authors have demonstrated a difference in resting POBF in relation to IOP, with a lower POBF in those with a higher IOP. ${ }^{8} 9$ Quaranta et al also demonstrated a lower POBF in normal tension glaucoma patients when compared to normals despite similar resting IOP and suggested these findings may be consistent with a lack of myogenic autoregulation in response to IOP induced modifications of the perfusion pressure in these patients. ${ }^{10}$ There are a number of reasons why we were unable to demonstrate significant differences among our groups and resting POBF although trends were apparentfirstly, because of the wide interindividual variation in normal values of resting POBF; larger groups may be required to clearly demonstrate these differences. We also recognise that there may be bias in the selection of our groups in that our normal tension group did not undergo phasing and were selected on a single measurement of Goldmann IOP. This was because it was the policy of the unit not to subject patients to phasing unless there was evidence of non-response to treatment; we wanted to capture patients before they were on any treatment. It is also apparent that there is a difficulty in classifying patients into a particular glaucoma group depending on the method of IOP measurement used as illustrated by the differences between the Goldmann and pneumotonometric IOP means shown in Table 1 . The groups were initially assigned according to the Goldmann measurement of IOP and the ranges and standard deviations demonstrate clear distinctions based on IOP between our groups; however, the pneumotonometric measurement is slightly different demonstrating some overlap between groups. This illustrates that some bias may be introduced by the method of IOP ascertainment in any study but we feel justified in selecting our groups based on the Goldmann IOP, this still being the "gold standard" of clinical measurement. We performed further analyses based on the pneumtonometric values because POBF is a function of IOP, therefore using the IOP from which the POBF is derived. The analysis of the pneumotonometric IOP does demonstrate a statistical difference in the means between the groups, suggesting the grouping is reasonably 
accurate. The analyses were also performed using the Goldmann IOPs and no other significant differences were revealed. Despite these possible flaws it is also likely that POBF is not sufficiently specific or sensitive enough to detect any important vascular differences between these groups of subjects.

The resting POBF was generally higher in females, a finding confirmed by other workers which has been attributed to the higher resting heart rate in females. ${ }^{911}$ Our study also reflects others' findings that increasing age is accompanied by a fall in resting POBF ${ }^{12}{ }^{13}$ To allow for these effects we adjusted for age and sex when comparing our three groups. We had intentionally excluded those with greater than 6 dioptres of refractive error as it has been shown that this, and more specifically axial length ${ }^{14}$ will influence POBF. Our study again reflects the findings of others of a trend towards a higher POBF in hypermetropes.

Overall, POBF fell during Valsalva. The greatest predictor of the fall, however, was the resting value of POBF, rather than the group. Studies of POBF in NTG patients have shown that a fall in POBF occurs when they adopt the supine position. ${ }^{15}$ Trew and Smith suggested the reason for this may be related to the fall in heart rate: if heart rate goes down POBF will fall because it is a measure of volume flow per minute. However, this can only partly explain differences in POBF, as illustrated by our study, for the heart rate rose in virtually all of our patients while performing Valsalva. In phase 2 of Valsalva, heart rate gradually rises so we have to assume that the single arterial pulse volume must fall. We reiterate that the pneumotonometer gives us a measurement only of the pulsatile component of ocular blood flow, and that there may be a shift from pulsatile to non-pulsatile flow during the Valsalva manoeuvre.

With respect to the association of certain risk factors we tried to use well recognised risk factors based on Drance et al's findings of factors involved in the production of normal tension glaucoma such as vasospasticity, to try and establish an association with $\mathrm{POBF}^{16}$ The division is somewhat arbitrary and although Schulzer found two distinct populations with vasospastic tendencies in one group and more generalised cardiovascular disease in the other he found that both high and normal tension glaucoma subjects were distributed evenly between these groups, ${ }^{17}$ whereas we demonstrated a statistically significant difference in the distribution of these types of risk factor between our groups. With the small numbers with clearly defined risk factors in this study we were unable to demonstrate an association between risk factor and change in POBF.

In summary, our study demonstrates trends between groups, with a smaller fall in POBF during Valsalva in the POAG group than the other groups but the strongest predictor of amount of change in POBF was the resting POBF. The Valsalva manoeuvre can be used to create alterations in ocular blood flow and causes a marked reduction in POBF in most patients. Unfortunately, because of the wide interindividual variability in measurements, POBF cannot be used to predict disease in an individual and it is not possible to say whether a greater change in POBF during Valsalva indicates a better or worse prognosis or whether our trumpet player should play less frequently.

\section{Authors' affiliations}

J Khan, AMD Study, Box 261, Addenbrooke's Hospital, Hills Road, Cambridge CB2 2QQ, UK

E H Hughes, University Division of Ophthalmology, Bristol Eye Hospital, Lower Maudlin Street, Bristol BS7 8EG, UK

B D M Tom , Centre for Applied Medical Statistics, Department of Public Health and Primary Care, University of Cambridge, Institute of Public Health, University Forvie Site, Robinson Way, Cambridge CB2 2SR, UK J P Diamond, Bristol Eye Hospital, Lower Maudlin Street, Bristol BSI 2LX, UK

Correspondence to: Jane Khan

Accepted for publication 29 April 2002

\section{REFERENCES}

1 Krakau CE. Calculation of the pulsatile ocular blood flow. Invest Ophthalmol Vis Sci 1992;33:2754-6.

2 Yang YC, Hulbert MF, Batterbury M, et al. Pulsatile ocular blood flow measurements in healthy eyes: reproducibility and reference values. J Glaucoma 1997:6:175-9.

3 Kothe AC. The effect of posture on intraocular pressure and pulsatile ocular blood flow in normal and glaucomatous eyes. Surv Ophthalmol 1994;38(Suppl):S191-7.

4 Korner PI, Tonkin AM, Uther JB. Reflex and mechanical circulatory effects of graded Valsalva maneuvers in normal man. J Appl Physiol 1976;40:434-40.

5 Schuman JS, Massicotte EC, Connolly S, et al. Increased intraocular pressure and visual field defects in high resistance wind instrument players. Ophthalmology 2000;107:127-33.

6 Lacey B, Rankin SA. The effect of increased intrathoracic pressure on pulsatile ocular blood flow. Invest Ophthalmol Vis Sci 1997;38:S1265.

7 Rafuse PE, Mills DW, Hooper PL, et al. Effects of Valsalva's manoeuvre on intraocular pressure. Can J Ophthalmol 1994;29:73-6.

8 Silver DM, Farrell RA, Langham ME, et al. Estimation of pulsatile ocular blood flow from intraocular pressure. Acta Ophthalmol 1989;191(Suppl): 25-9

9 Fontana L, Poinoosawmy D, Bunce CV, et al. Pulsatile ocular blood flow investigation in asymmetric normal tension glaucoma and normal subjects [see comments]. Br J Ophthalmol 1998:82:731-6.

10 Quaranta L, Manni G, Donato F, et al. The effect of increased intraocular pressure on pulsatile ocular blood flow in low tension glaucoma. Surv Ophthalmol 1994;38(Suppl):S177-81.

11 Trew DR, James CB, Thomas $\mathrm{SH}$, et al. Factors influencing the ocular pulse-the heart rate. Graefes Arch Clin Exp Ophthalmol 1991;229:553-6.

12 Ravalico G, Pastori G, Croce $M$, et al. Pulsatile ocular blood flow variations with axial length and refractive error. Ophthalmologica 1997:211:271-3

13 Dallinger S, Findl O, Strenn K, et al. Age dependence of choroidal blood flow. J Am Geriatr Soc 1998;46:484-7.

14 Mori F, Konno S, Hikichi T, et al. Factors affecting pulsatile ocular blood flow in normal subjects. Br J Ophthalmol 2001;85:529-30.

15 Trew DR, Smith SE. Postural studies in pulsatile ocular blood flow: I. Ocular hypertension and normotension. Br J Ophthalmol 1991;75:66-70.

16 Drance SM, Sweeney VP, Morgan RW, et al. Studies of factors involved in the production of low tension glaucoma. Arch Ophthalmol 1973;89:457-65.

17 Schulzer M, Drance SM, Carter CJ, et al. Biostatistical evidence for two distinct chronic open angle glaucoma populations. $\mathrm{Br} J$ Ophthalmol 1990;74:196-200 